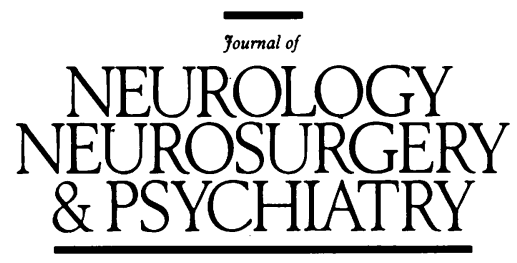

Editorial

\title{
What is neuropsychiatry?*
}

Neuropsychiatry is a rather seductive term. It implies an amalgam between neurology and psychiatry, which in a sense it is. But there is a great deal more to the name and to the discipline than that. If we view it too narrowly as an admixture of neurology and psychiatry we miss out on the essence of the subject as an important and developing branch of psychiatry.

So what precisely is it? The matter was debated at the summer meeting of the British Neuropsychiatry Association, held in conjunction with the sister organisation, the American Neuropsychiatric Association. As a prelude to discussion a detailed questionnaire had been distributed to members of both organisations.

First, it was clear that the term was seen as representing an approach to the study of psychiatric disorders, rather than merely specifying the study of a restricted range of conditions. Indeed some two thirds of respondents felt that all psychiatric disorders could be approached from the viewpoint of a possible neurological basis. The Americans were bolder in this than the British, and neurological respondents more certain than psychiatrists. As for the subset of psychiatric disorders most directly of concern to neuropsychiatry, this was variously defined as "those for which knowledge of brain structure and function is indispensable", or "those with a well-defined cerebral basis". Furthermore, virtually all agreed that neuropsychiatry was concerned with psychiatric aspects of neurological disorders, and that it dealt with the borderland territory between clinical neurology and clinical psychiatry.

In other words neuropsychiatry is seen by these Associations as constituting a particular approach to the understanding of psychiatric disorder and as having relevance to a wide range of such disorders, but with its own special field within the clinical domain.

\section{Evolution}

Before proceeding further it may be useful to consider the origin and evolution of the subject.

Psychiatry started much earlier than neurology, when in the 17 th century medicine took over from the church a role in the care of the insane. From the very beginning psychiatry dealt with a class of persons, namely, the "mentally ill". Its clients were very heterogeneous, suffering from all manner of conditions. And for a couple of centuries psychiatry was wildly adrift in its attempts to formulate theories about what was amiss with them. Neurology came into being much later in the first part of the nineteenth century, taking as its point of origin diseases of the brain. It developed from the start as a rational and scientific discipline, tying certain maladies to the growing knowledge of brain function and brain organisation.

Still very unsure of itself, psychiatry watched from the sidelines and envied this success. So the intimate tie between neurology and neuropathology became an avenue for exploring psychiatric disorders. Many of the great German psychiatrists of the nineteenth century were at the same time neuropathologists. Resounding successes were achieved for example, in the field of the dementias and general paresis. Lesions could at last be displayed in the brains of certain psychiatric patients.

This was in a sense a heyday for neuropsychiatry, with both neurology and psychiatry employing parallel approaches. Many clinical practitioners were indistinguishably neurologists and psychiatrists, a true amalgam of both. Further developments, however, served to disrupt the status quo. Chief among these was the growth of psychodynamics, a system of thinking about mental disorder which had little to do with the brain. It enriched psychiatry, but meant nothing to neurology, and it drove a wedge between the two disciplines. Psychiatrists were keen to exploit these new approaches, and neurologists became glad to leave the muddle of mental illness to others. Later we saw the rise of social and epidemiological psychiatry, again largely devoid of relevance to the brain, yet still providing psychiatrists with new insights into their work. Multi-factorial approaches to mental disorder prospered. The neuropsychiatric view came often to be dubbed narrow, old fashioned, and atherapeutic.

Some, however, remained loyal all through to the concept of neuropsychiatry. Sir Charles Symonds, the doyen of British neurologists, was a fighter for the cause, regretting psychiatry's drift away from neurology and neuroscience, and making some remarkable recommendations. ${ }^{1}$

Towards the end of the Second World War the Royal College of Physicians set up a committee to consider psychological medicine. Symonds, with Riddoch, wrote a minority report, urging that general hospitals should set up Neuropsychiatry Clinics as the focus for the work of psychiatrists, neurologists and neurosurgeons side by side. The report noted the wide overlap between all three disciplines, the common use of investigative techniques, and the interweaving of research interests. The directors of such clinics could be variously psychiatrists, neurologists or neurosurgeons. Psychiatrists and neurologists should have common basic training with a grounding in general medicine, then three years divided between psychiatry and 
neurology along with the basic sciences appropriate to both. A final two years would be devoted to one or the other discipline alone.

But this view did not prevail. The report was rejected by the physicians, and the Association of British Neurologists was equally alarmed at the prospects. The neurologists overwhelmingly refused to enter into any formal alliance with psychiatrists. In the subsequent years, neurologists and psychiatrists have followed very different paths, with a steady erosion of most of the links in training requirements. The brave new world which was briefly urged in the post-war years simply did not come into being.

So what is the status of present day neuropsychiatry? It is perhaps best to consider two aspects separately; the clinical - what neuropsychiatrists do, and the academic an approach to mental disorder with research and philosophical implications.

\section{Academic neuropsychiatry}

To consider the latter first, neuropsychiatry may be regarded as that aspect of psychiatry which (like neurology) seeks to advance understanding of clinical problems through increased knowledge of brain structure and function. It fundamentally involves the understanding of brain mechanisms in relation to mental disorder. This may be widely applied so that schizophrenia, for example, now falls well within its compass.

In these respects neuropsychiatry is the counterpart of neurology - the part of psychiatry that stayed in league with it when the main corpus of psychiatry did not. It is an approach which sees neurological and neuropathological knowledge as very important. And it seizes equally eagerly on the more modern disciplines of neuropsychology, neurochemistry, immunology and molecular genetics. In brief, it prospers from every science that can teach us about the brain.

This, if you like, is the basis of neuropsychiatry. But it must be stressed that its practitioners are psychiatrists with a grasp of other approaches as well. They will not, or should not, be narrowly confined by their interest in the brain. Social, developmental, psychodynamic and interpersonal forces must also be considered in parallel with the above if we are to grapple successfully with some of the enigmas of psychiatric disorder. Neuropsychiatry should not be seen as an all-exclusive domain.

One must also take issue with those who claim that the neuropsychiatric approach can account for all mental disorder. Such vaulting ambition is reminiscent of the once proud claims of psychodynamic theory. Human beings are more complex than that, not least because of their insistence on interacting with one another. In some cases, perhaps, the environment will be found to hold more pathology than does the brain itself. Neuropsychiatry is best seen as standing shoulder to shoulder with other approaches, one perspective of several, and with closer applicability to some forms of mental illness than others.

\section{Clinical neuropsychiatry}

With regard to clinical practice the emphasis will clearly be more on certain disorders than others as behoves an interest in the brain. In large part therefore neuropsychiatrists will deal with those mental disorders which can be demonstrated to owe their origins to brain malfunction of a clearly identifiable nature.

Around this there will be various important penumbras. Thus in some cases the brain malfunction will be less than immediately obvious, and the neuropsychiatrist will be called on to adjudicate. Sometimes it will be quite unclear whether the patient falls into the organic or the nonorganic camp, hence the importance of a thorough
Table Diagnostic categories among patients with known organic psychosyndromes referred to the Maudsley neuropsychiatric clinic

Dementia, presenile and senile

Sequelae of head injury

Alcoholism (Korsakoff's syndrome, dementia, psychoses)

Memory disorders

Brain developmental abnormalities

Epilepsy

Confusional states

Toxic disorders (additional to alcohol)

Cexic disorders

Others (general paresis, sarcoid of CNS, etc)

acquaintance with all forms of mental disorder. And because the neuropsychiatrist works closely alongside neurological colleagues he or she will see the manifold emotional and other complications that can arise in neurological patients.

By way of illustration, the last 300 patients referred to the Maudsley neuropsychiatric clinic have been analysed. Almost half $(49 \%)$ were suffering from clear organic psychosyndromes, referred for closer diagnosis, treatment, or advice. The conditions responsible are shown in decreasing order of numbers in the table. Epilepsy features relatively rarely because of the coexistence at the Maudsley of a specialised clinic for patients with epilepsy.

Next in frequency (22\%) were patients with established psychiatric illnesses (schizophrenic, affective or neurotic) in whom some reason had arisen to suspect brain malfunction. This mostly derived from the mental state (impaired cognition, abnormalities of speech, visual hallucinations), or from abnormal results on neuroimaging or electroencephalography. Failures of treatment response were quite often the reason for referral, likewise abnormalities on physical examination, mostly motor disorder.

Third in frequency $(20 \%)$ were a group of patients whose symptoms gave rise to uncertainty about organic or non-organic disorder - patients with possible pseudodementia, pseudoseizures or suspected hysterical conversion disorder. Sleep abnormalities included hypersomnia, insomnia and hallucinatory states. Speech disorders included mutism and uncertainties between dysphasia and schizophrenic speech disorder. The remaining $10 \%$ of patients were referred from neurological colleagues (such as, patients with Parkinson's disease, multiple sclerosis, cerebrovascular accidents) with the expected range. of cognitive impairments, depression and anxiety. Occasional patients had shown psychotic developments.

This represents the practice of one neuropsychiatry clinic. It illustrates the range, and the breadth, of diagnostic possibilities which must often be kept in mind.

Neuropsychiatry in relation to other subspecialties

Finally, one may consider the links with other subspecialities - biological psychiatry, liaison psychiatry, and behavioural neurology. All have their champions and their own peculiar histories. It is difficult to disentangle the threads.

Biological psychiatry seems to have grown from the start with biologically oriented treatment strategies. Certainly from the 1950 s onwards pharmacological discoveries began to transform psychiatric practice, and their successes led on to an intensive focus on brain biochemistry. Discoveries in neuroendocrinology were further appended, knowledge of cell mechanisms grew, and finally we have witnessed the achievements of molecular genetics.

Biological psychiatry exploits this new knowledge to advance understanding of mental illness. But it differs from neuropsychiatry in two important respects. First, it is 
concerned with events predominantly at the microscopic or cellular level. The biological psychiatrist can prosper while knowing little about brain organisation more broadly, and while having little interest in neurology or neuropsychology. That is far from true of the neuropsychiatrist who remains fascinated by neural systems, brain organisation, the functions of different parts of the brain and their relationships with one another. Put very baldly, the fundamental sciences for neuropsychiatry (and neurology) are neuroanatomy and neurophysiology; those for biological psychiatry centre on the cell and its chemical processes.

A second difference derives from the above. The neuropsychiatrists have their clinical base, a territory within psychiatry in which he or she is acknowledged to excel. The biological psychiatrist does not have this circumscribed clinical field. Biological psychiatry is essentially an academic approach, applied widely to all fields of psychiatric disorder; the neuroses, the psychoses and the personality disorders all come within its remit.

The two are of course interwoven. Neuropsychiatrists (and neurologists) are also interested in the cell and in chemical processes. They too employ pharmacological treatments. So one wonders why the new term "biological psychiatry" was coined? Perhaps it was in reaction to the close links between neuropsychiatry and neurology - a reflection of the new psychiatry's proud independence. Or perhaps it was felt that traditional neuropsychiatry was tending to lag behind in its appreciation of the significance of the new neurochemical and other discoveries.

At the moment neuropsychiatry and biological psychiatry stand in a somewhat uneasy relationship with one another. One even wonders whether the one will come to subsume the other. The questionnaire mentioned earlier sought to address this issue, one question stating that biological psychiatry included neuropsychiatry, the other stating the reverse. Overall, the replies were remarkably evenly balanced, but differences emerged between the UK and the USA. The UK psychiatrists favoured the ascendancy of biological psychiatry over neuropsychiatry to a statistically significant extent (even though they had joined the British Neuropsychiatry Association!). The USA psychiatrists were more resolute in championing neuropsychiatry over biological psychiatry. Neurologists from both countries also tended to see neuropsychiatry as encompassing biological psychiatry. However, a substantial $10 \%$ of respondents admitted uncertainty. The situation is clearly in a state of flux.

Liaison psychiatry is an important and thrusting force in British psychiatry. Its place is clearer to discern. Liaison psychiatrists work in general hospitals, and see referrals from their medical and surgical colleagues. They deal of course with neuropsychiatry to the extent that they work alongside neurologists and neurosurgeons. But their brief is broad and their field heterogeneous - some specialise in liaison referrals from renal units, gastroenterologists, oncologists, or in relation to obstetrics and gynaecology. Liaison psychiatry is a practical clinical discipline, rather than a specialised theoretical approach.

Behavioural neurology has had wider development in America than the UK and has grown essentially from neurology. Its emergence may have reflected dissatisfaction with an earlier era of psychiatric practice in the USA. Behavioural neurology looks at abnormal behaviour through the eyes of the neurologist, using neurological knowledge in attempts at explanation. The focus is on defined areas of abnormal behaviour or experience, rather than on broad segments of mental disorder. Models are derived from lesion studies and knowledge of brain organisation, often resulting in theories of great interest. For example, we have models of personality dysfunction based on temporo-limbic hyperconnection, and of obsessional neurosis based on striate-frontal imbalance. Analogies are drawn between depressive psychomotor retardation and subcortical dementia. It would seem, however, that behavioural neurology tends to give an incomplete picture, since its view of behaviour is bound to be narrow. The other forms of explanation available to psychiatrists are in the main left out of account.

The questionnaire asked whether neuropsychiatry was synonymous with behavioural neurology and drew a markedly negative response. It was, however, twice as often endorsed by neurologists as by psychiatrists both in the UK and the USA, with positive scores of $29 \%$ and $15 \%$ respectively.

In conclusion, these various subspecialities may be viewed as a complex amalgam, a dynamic field of interrelationships between various viewpoints and areas of knowledge. It should not be surprising that the present multifarious state has evolved, in our attempts to unravel something as complex as human mental disorder in relation to brain malfunction.

Neuropsychiatry itself has obviously had a long and complex history. It appears to survive very well as a vigorous branch of psychiatry despite the divisions of opinion that it has generated along the way. Fortunately the practitioners of neuropsychiatry have strong allies in their colleagues in neurology, neuropsychology and neuropathology, and in their links with workers in the laboratory neurosciences.

Institute of Psychiatry,

W A LISHMAN De Crespigmy Park, Denmark Hill, London, UK

«Based on a lecture given at the British Neuropsychiatry Association Summer Meeting, St Catherine's College, Oxford, 12-14 July 1992.

1 Symonds CP. Tria functa in Uno. The Sixth Sir Hugh Cairns Memorial Lecture. Addressed to The Society of British Neurological Surgeons, 25 September 1970. Oxford: Seacourt Press: Oxford, 1970. 